Predicting the Operational Acceptance of Route Advisories

\author{
Antony Evans (Crown Consulting, Inc.) \\ Paul Lee (NASA Ames)
}




\section{Reroute Advisories}

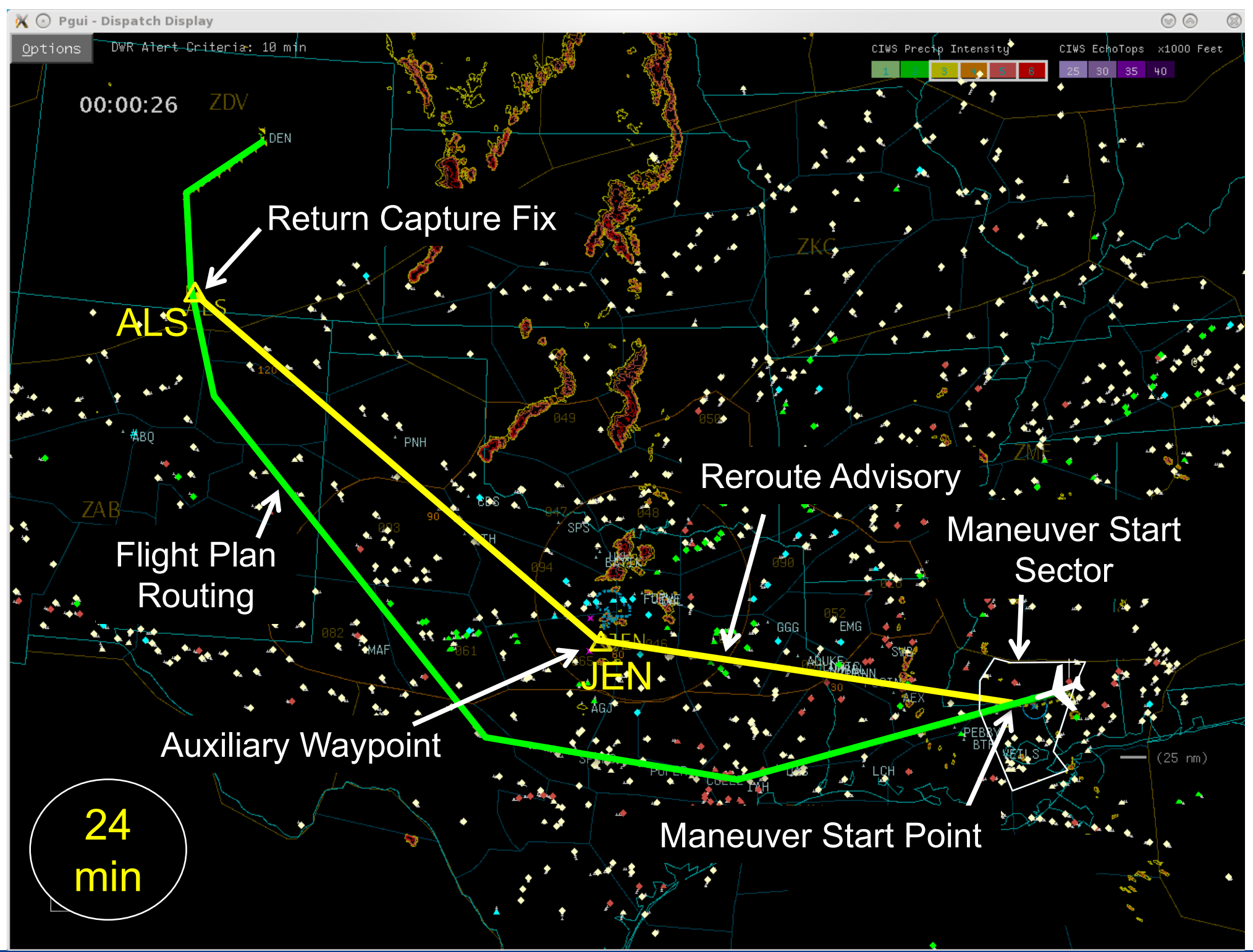




\section{Dynamic Weather Routes (DWR)}

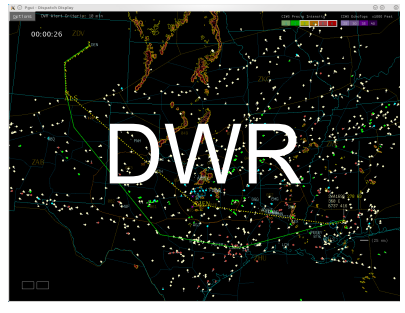

$\checkmark$

ATC Coordinator

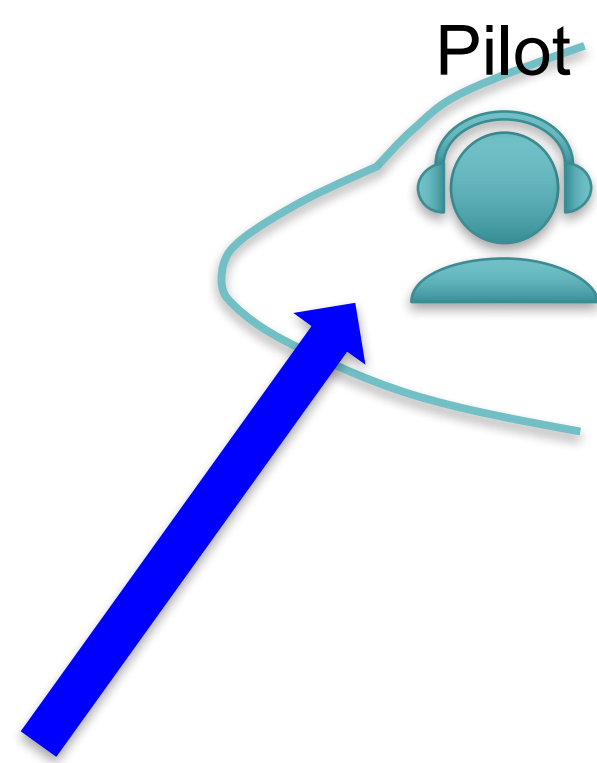

Dispatcher

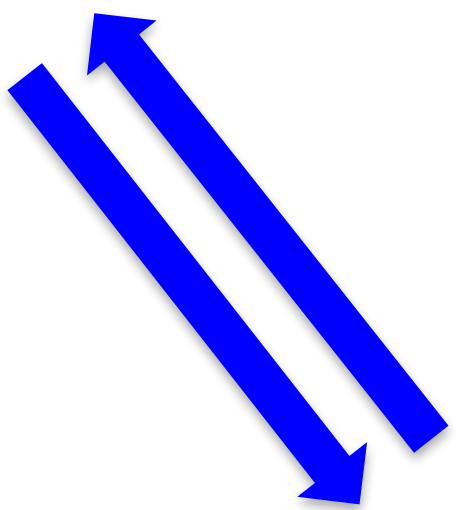

Air Traffic Controller
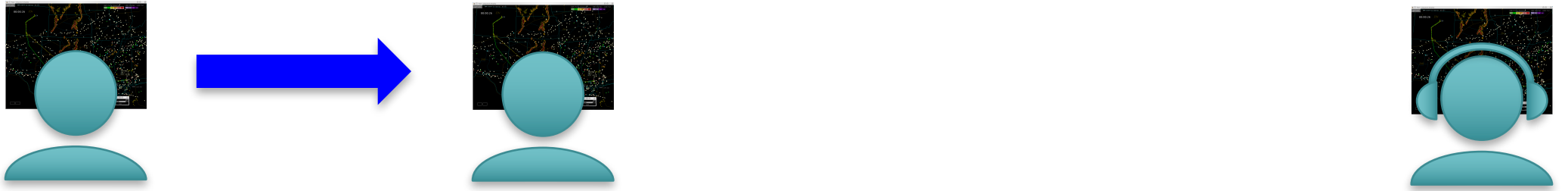


\section{Motivation}

ATC Response to DWR Route Advisory (DWR trial, 2014)

Route Observed in Flight Plan Amendment Data (Jun-Aug 2015)

\begin{tabular}{l|c|c|}
\hline & True & False \\
\hline Accepted & $97 \%$ & $3 \%$ \\
\hline Rejected & $69 \%$ & $31 \%$ \\
\cline { 2 - 3 }
\end{tabular}

Evans, et al. (2016)

- Historical usage required for ATC route acceptance

- Other factors also contribute to ATC acceptance

- Objective: Develop a predictor of operational acceptability for route advisories 


\section{Approach}

Identify features

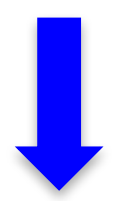

Extract data

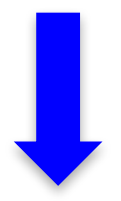

Data Mining

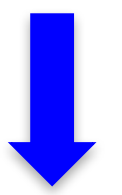

Validation
- Literature review

- Subject matter experts

- ATC accepted routes

- ATC rejected routes

- Binary classifier 


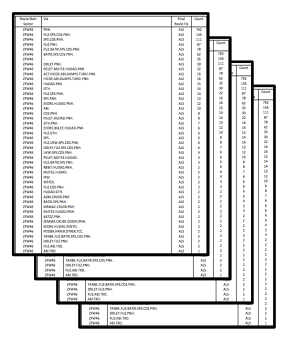

\section{Features - Number sectors over capacity Max demand to capacity ratio}

- Historical count (full route)

- Historical count (by segment)

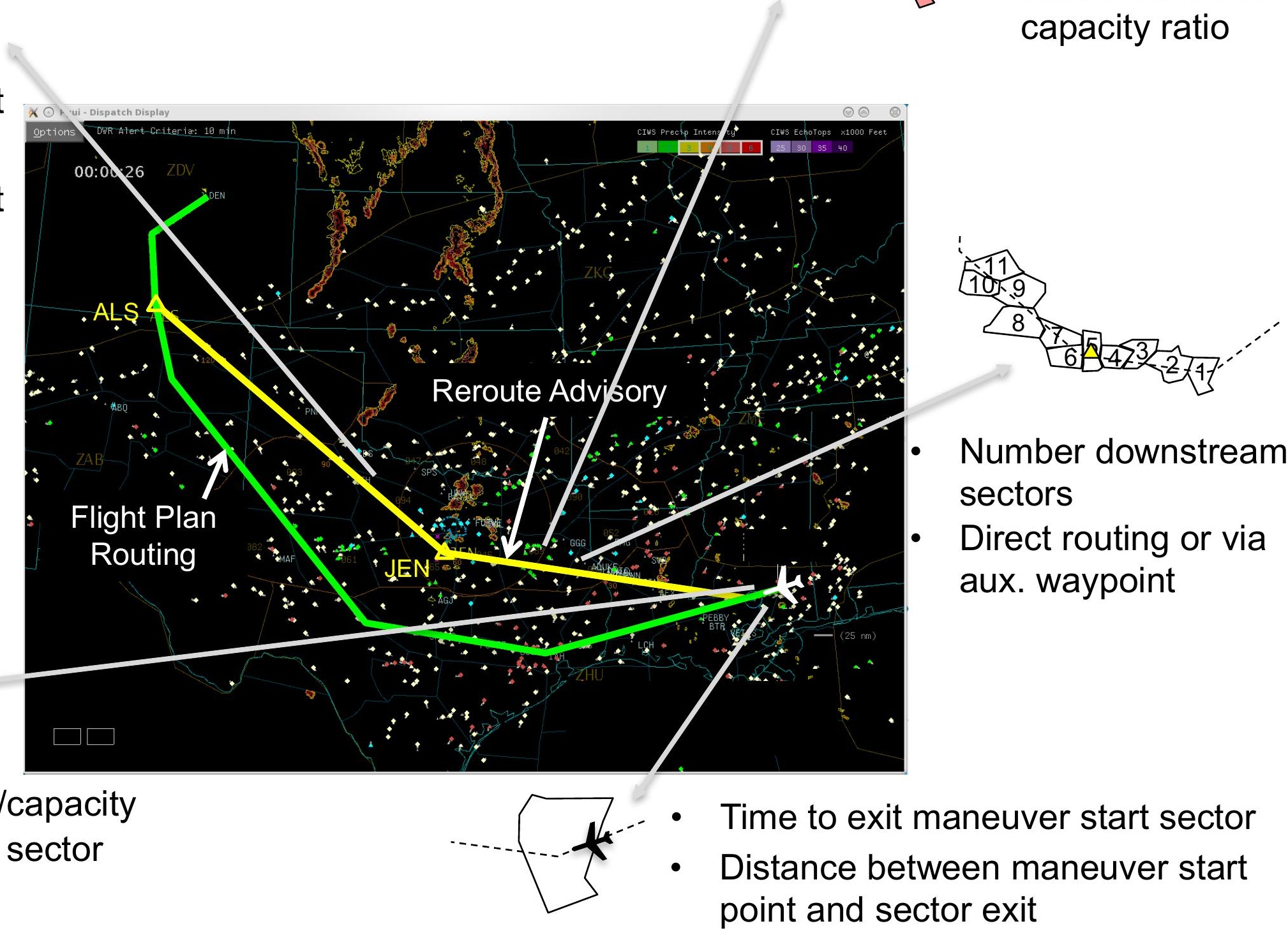




\section{Data}

Usage

ASDI Data

\begin{tabular}{c|c|c} 
June & July & August \\
2015 & 2015 & 2015 \\
\hline
\end{tabular}

Flight Plan Amendments
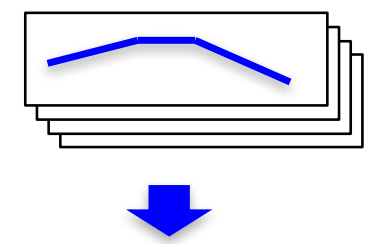

Common Routing Tables

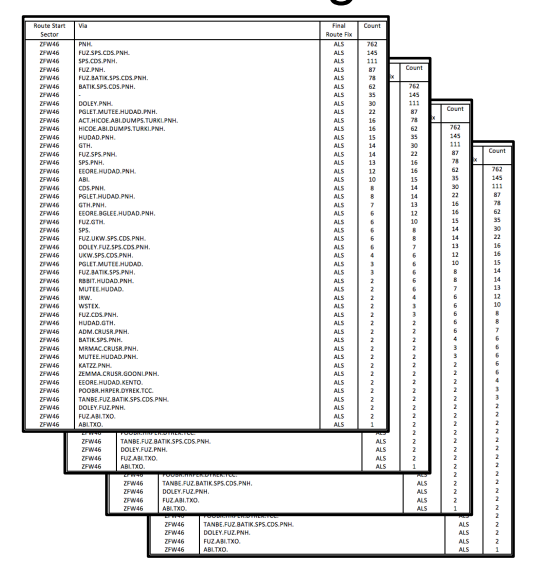

Feature Extraction

DWR Trial Data (ZFW and adjacent Centers)

\begin{tabular}{cccccc} 
May & June & July & August & September \\
2014 & 2014 & 2014 & 2014 & 2014 \\
\hline
\end{tabular}

DWR Advisories Flight Plan Amendments
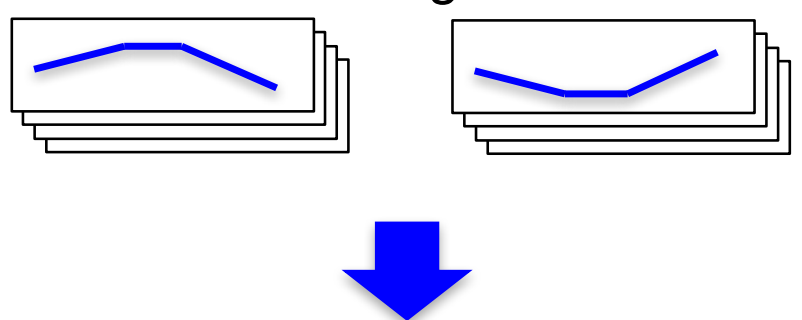

Future ATM Concepts Evaluation Tool (FACET)

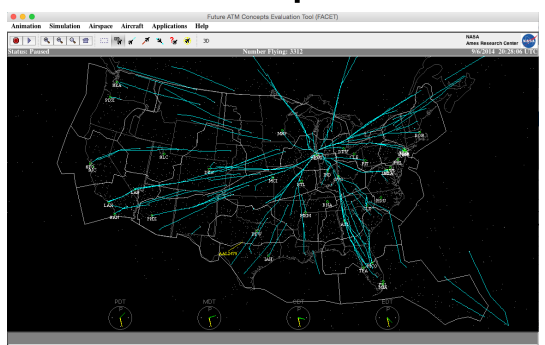

Sector Counts etc. 


\section{Classifying Routings}

DVC flight plan amendment implemented within $30 \mathrm{~min}$
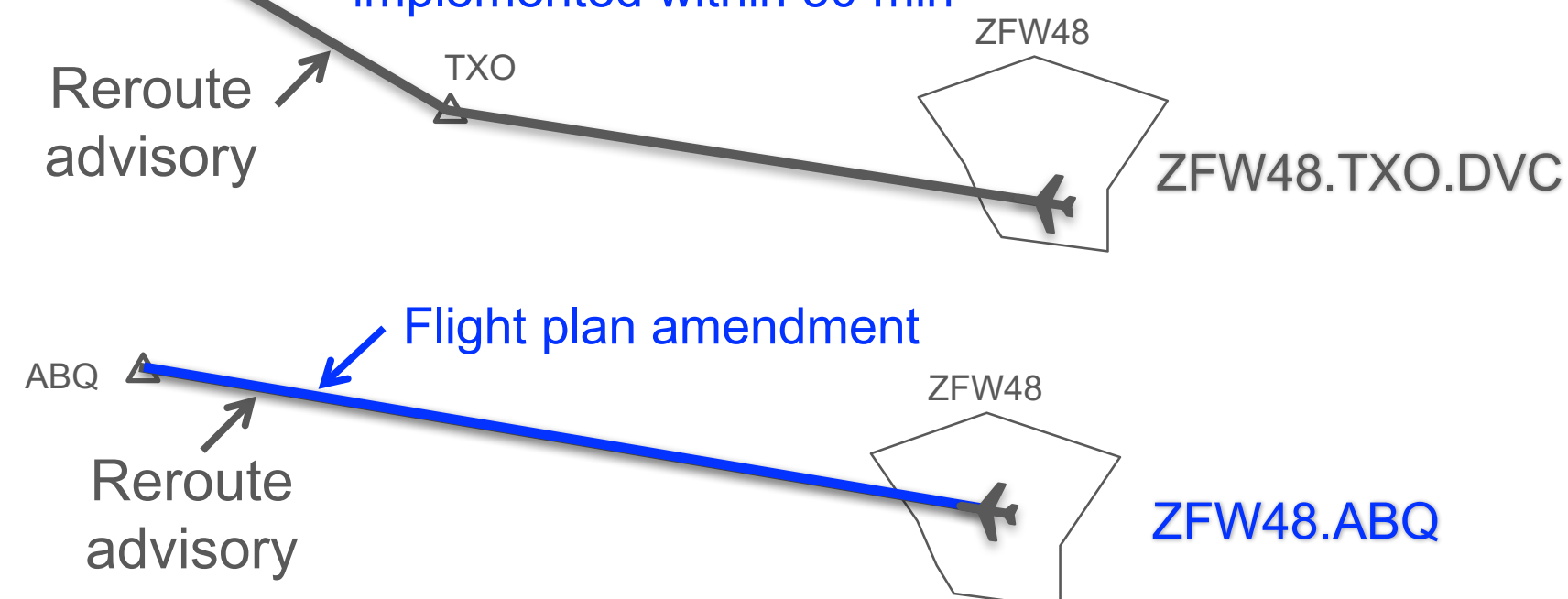

$\Delta^{A L S}$

Flight plan amendment

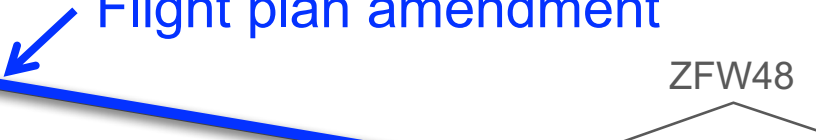

\section{ZFW48.ABQ}

ATC Acceptable

$(13 \%)$

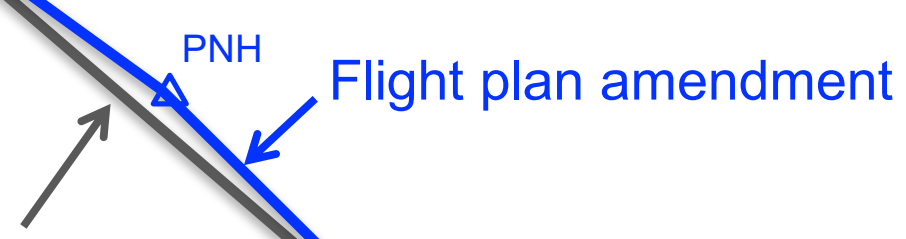

Reroute advisory

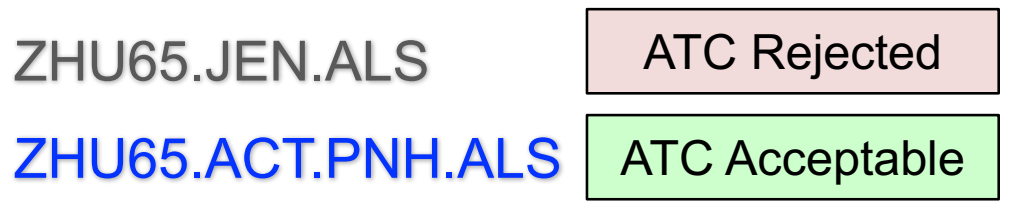




\section{Model Development}

Feature Selection

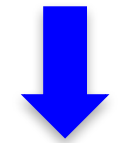

Model Selection

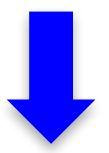

Parameter Selection

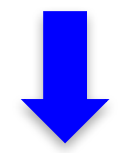

Model Validation
- Forward search through 10 features

- Logistic regression

- Decision tree

- Support Vector Machine (SVM) with sigmoid kernel

- Random forest

- Adaptive Boost (AdaBoost)

- e.g., Number of trees

- Nested cross-validation 


\section{Feature Selection}

- Forward Search with Random Forest, 10 -fold cross validation

- 317 to 544 observations - $40 \%$ to $48 \%$ Rejected; $60 \%$ to $52 \%$ Accepted
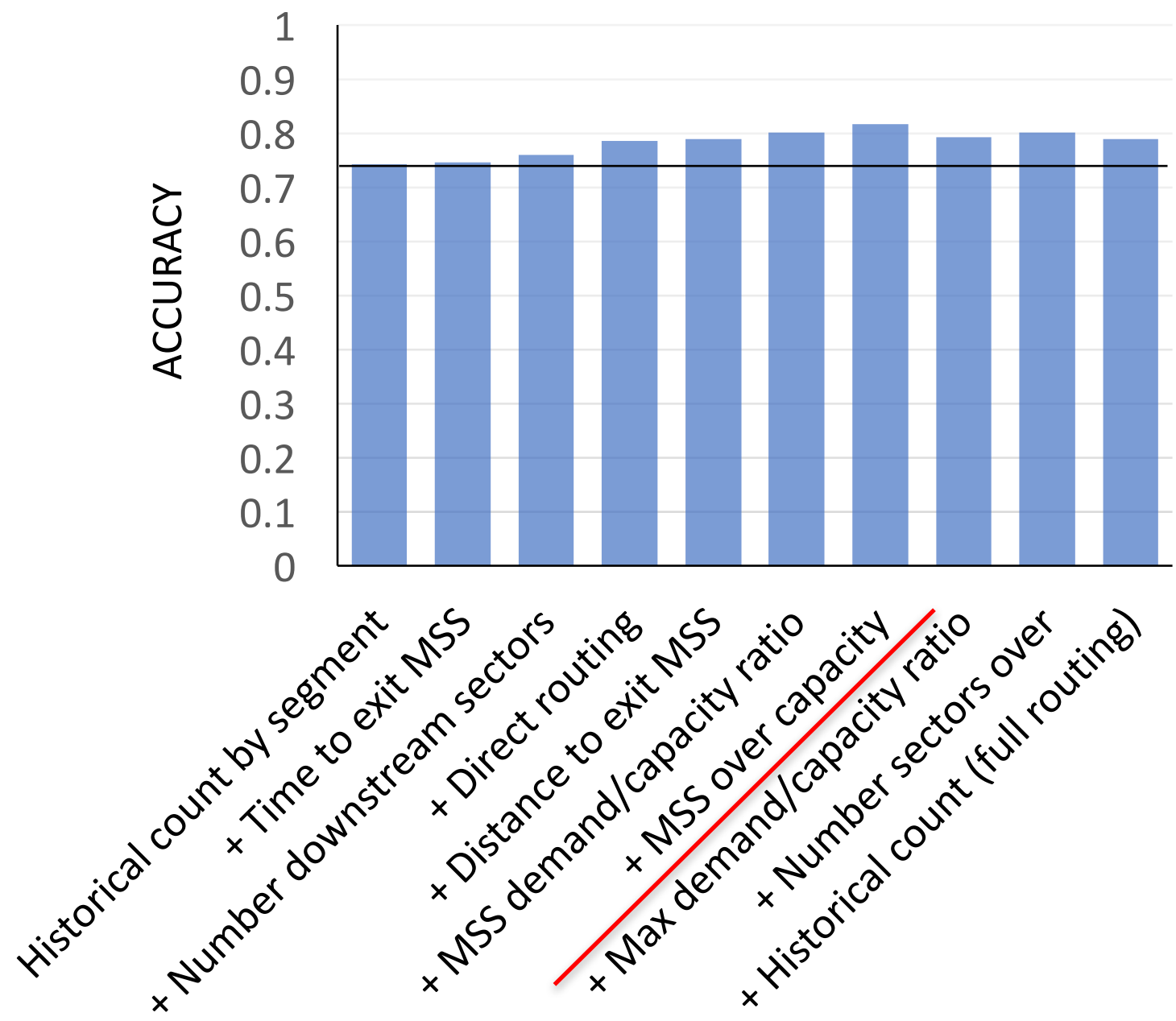


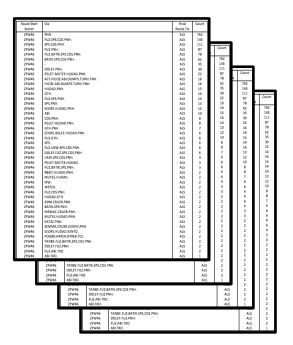

\section{Features

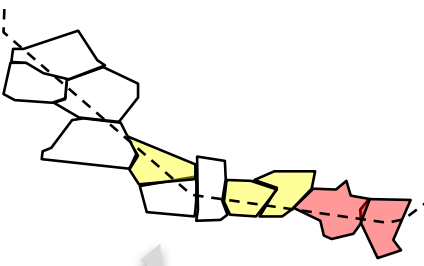

Number sectors - vver capacity Max demand to capacity ratio

Historical count (full route) Aistorical count (route segment)

Maneuver start

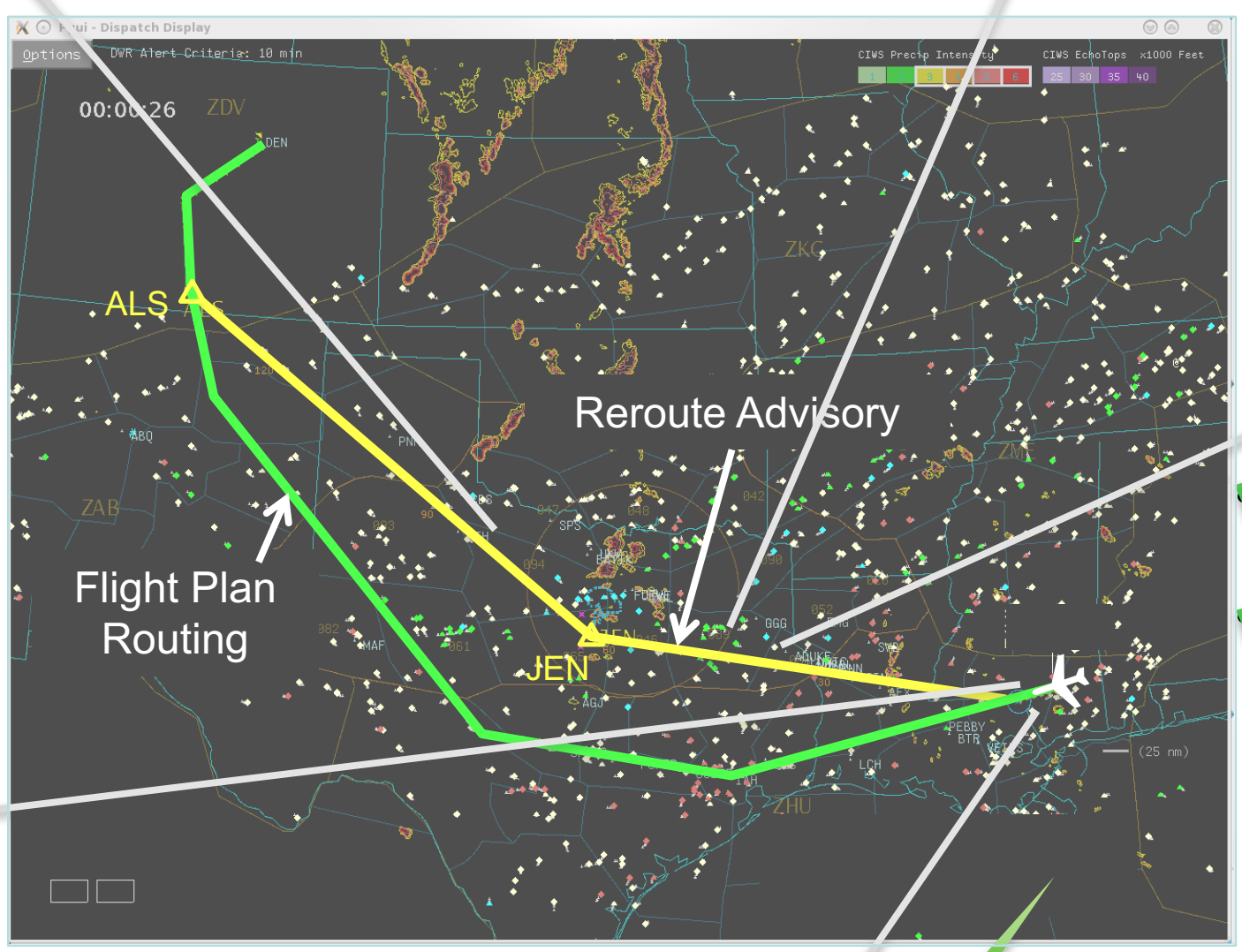

i11

109.9 $87-42 \times-2 \times 4$

Number downstream sectors

Direct routing or via aux. waypoint

sector demand/capacity Maneuver start sector over capacity

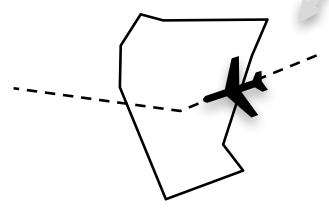

Tirne to exit maneuver start sector Distance between maneuver start point and sector exit 


\section{Model Selection}

- 7 features

- 10-fold cross validation

- 317 observations - 48\% Rejected; 52\% Accepted

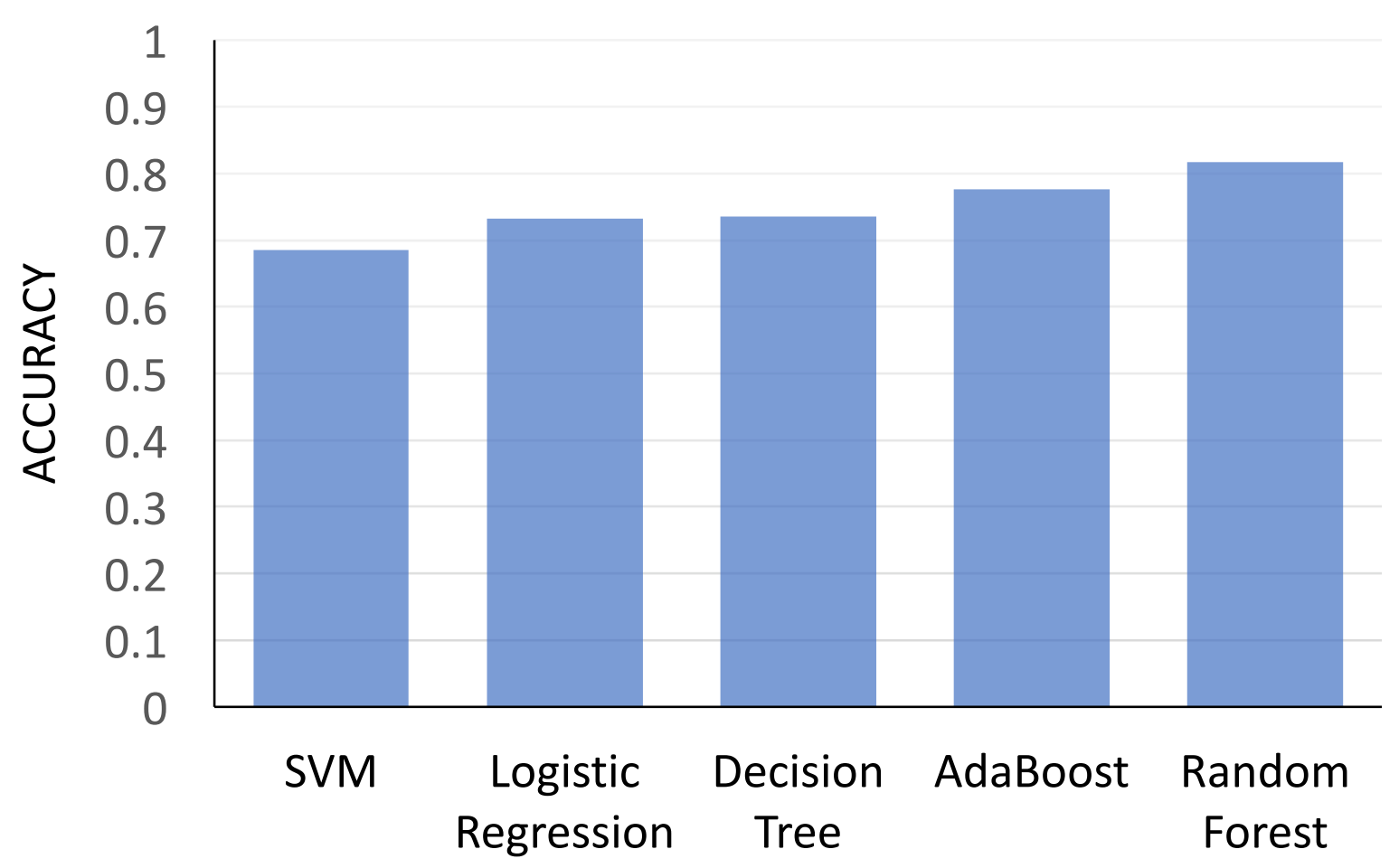

- Parameter Selection: 40 trees 


\section{Model Validation}

- 7 features, Random Forest, 40 trees

- Nested 10-fold cross validation

- 317 observations - 48\% Rejected (positive); 52\% Accepted (negative)

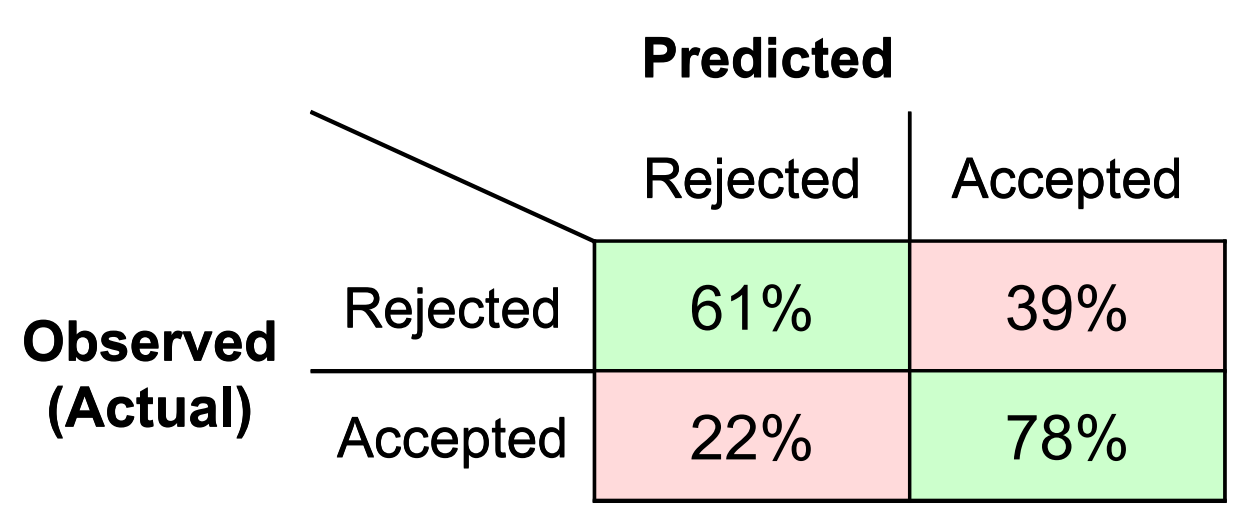

Model Accuracy: $70 \%$
Receiver Operating Characteristic Curve

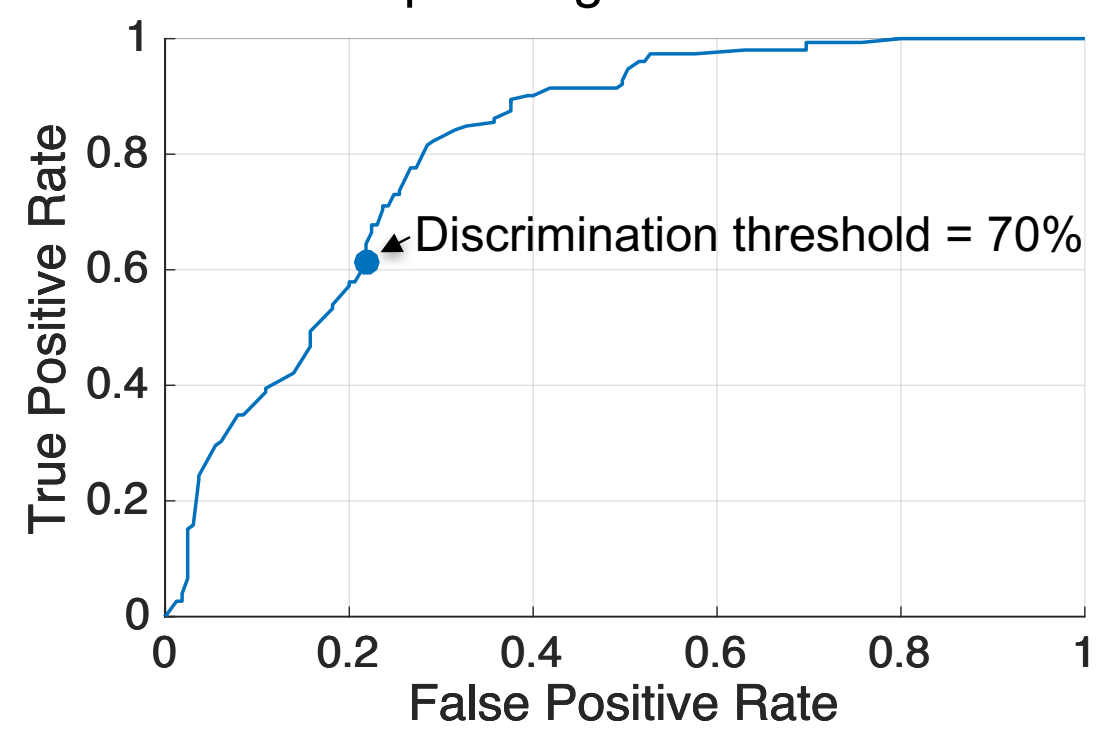




\section{Conclusions}

- Developed a predictor of operational acceptability for route advisories:

- Accuracy of $74 \%$

- Route rejection predicted at rate of $88 \%$

- Relevant model features:

- Historical usage

- Timing/location of request in maneuver start sector

- Number of downstream sectors

- Direct routing or via auxiliary waypoints

- Demand to capacity levels in maneuver start sector

- Best performing model is Random Forest with 40 trees 


\section{Future Work}

- Extension to other airspace

- Trial data for NAS Constraint Evaluation and Notification Tool (NASCENT)

- Improve features

- Include weather impact on maneuver start sector capacity

- Add other features, e.g., Center information

- May use voice recordings to identify timing and details of pilot requests to $A T C$ 
Questions? 


\section{Back-up Slides}




\section{DWR Use and Estimated Actual Savings}

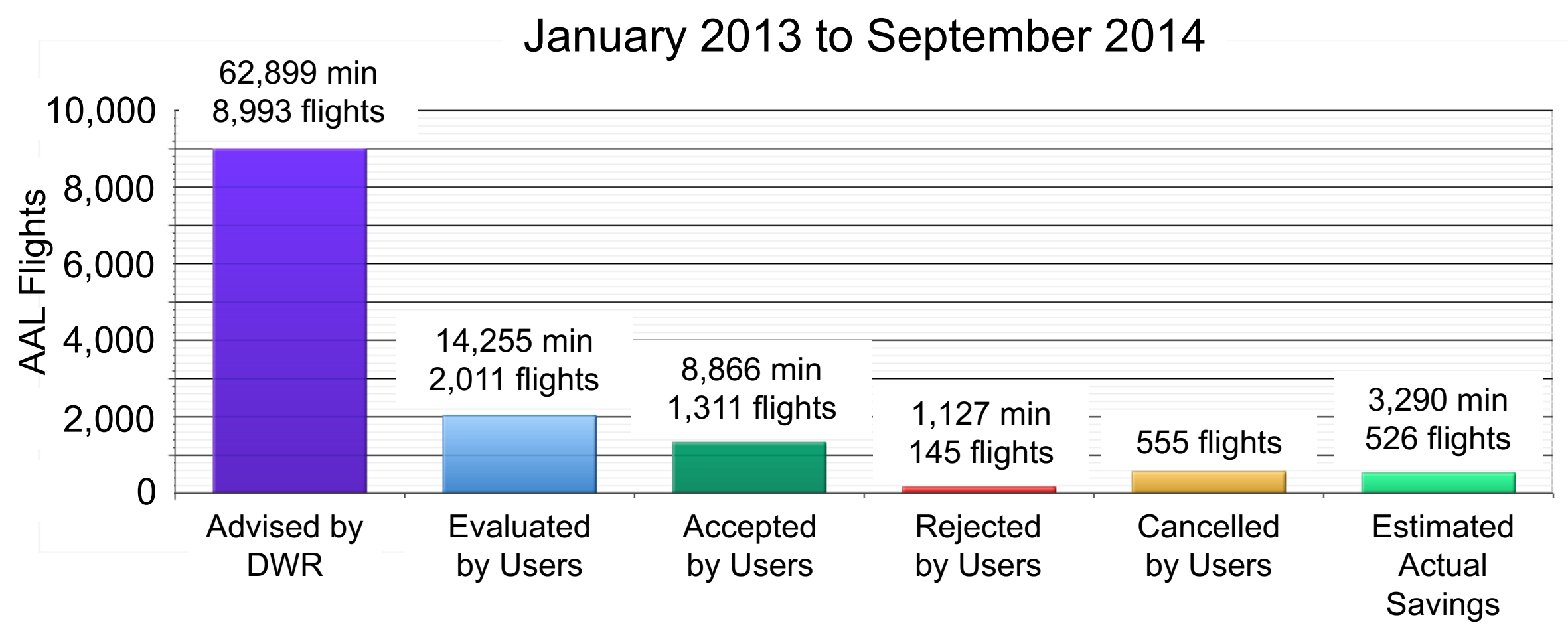

$40 \%$ of dispatcher accepted routes see actual savings

McNally, D., Sheth, K., Gong, C., Sterenchuk, M., Sahlman, S., Hinton, S., Lee, C., Shih, F-T., "Dynamic Weather Routes: Two Years of Operational Testing at American Airlines," Air Traffic Control Quarterly, Vol. 23, No. 1, pp. 55-81, 2015. 


\section{Traditional Model Development}

Dataset

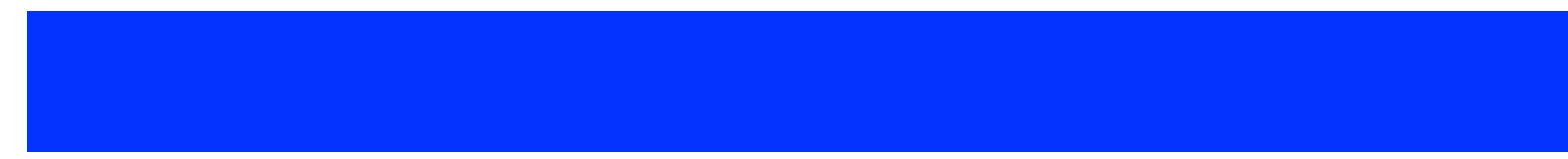




\section{Nested Cross Validation}

\section{Dataset}
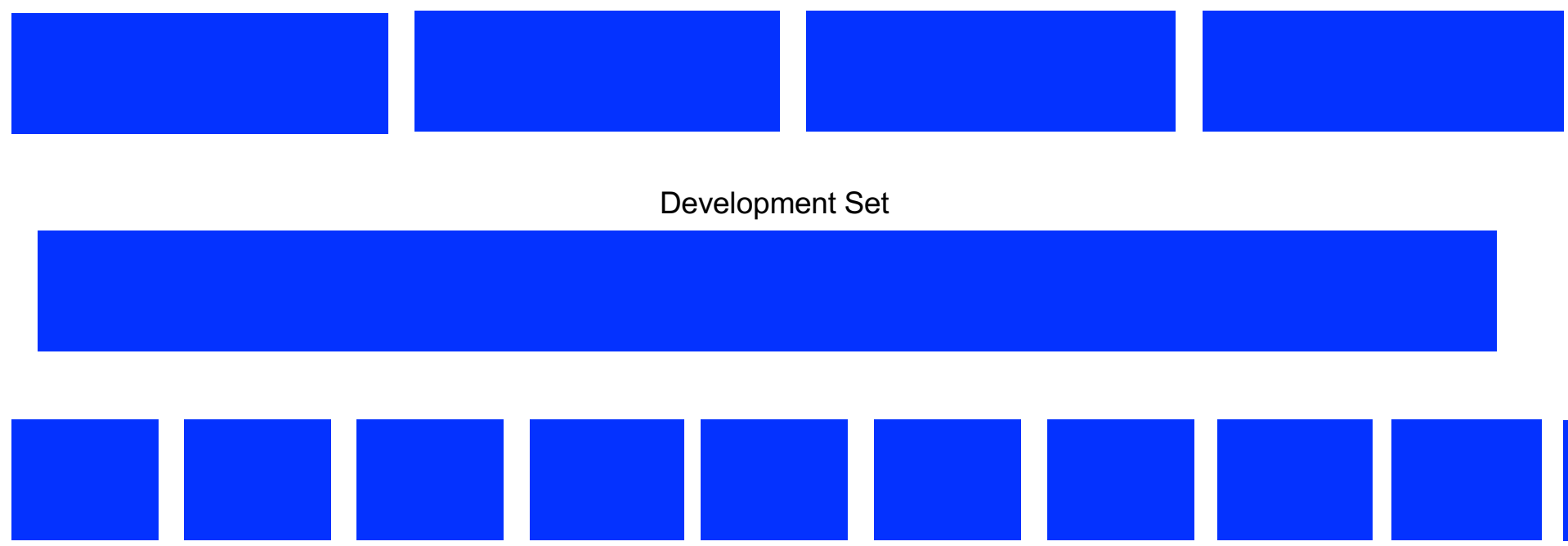

Training Set

Test Set 


\section{Feature Selection: Forward Search}

- Random Forest, 10-fold cross validation

- 317 to 544 observations $-40 \%$ to $48 \%$ Rejected (positive); $60 \%$ to $52 \%$ Accepted (negative)

F-Score $(50 \%$ discrimination threshold)

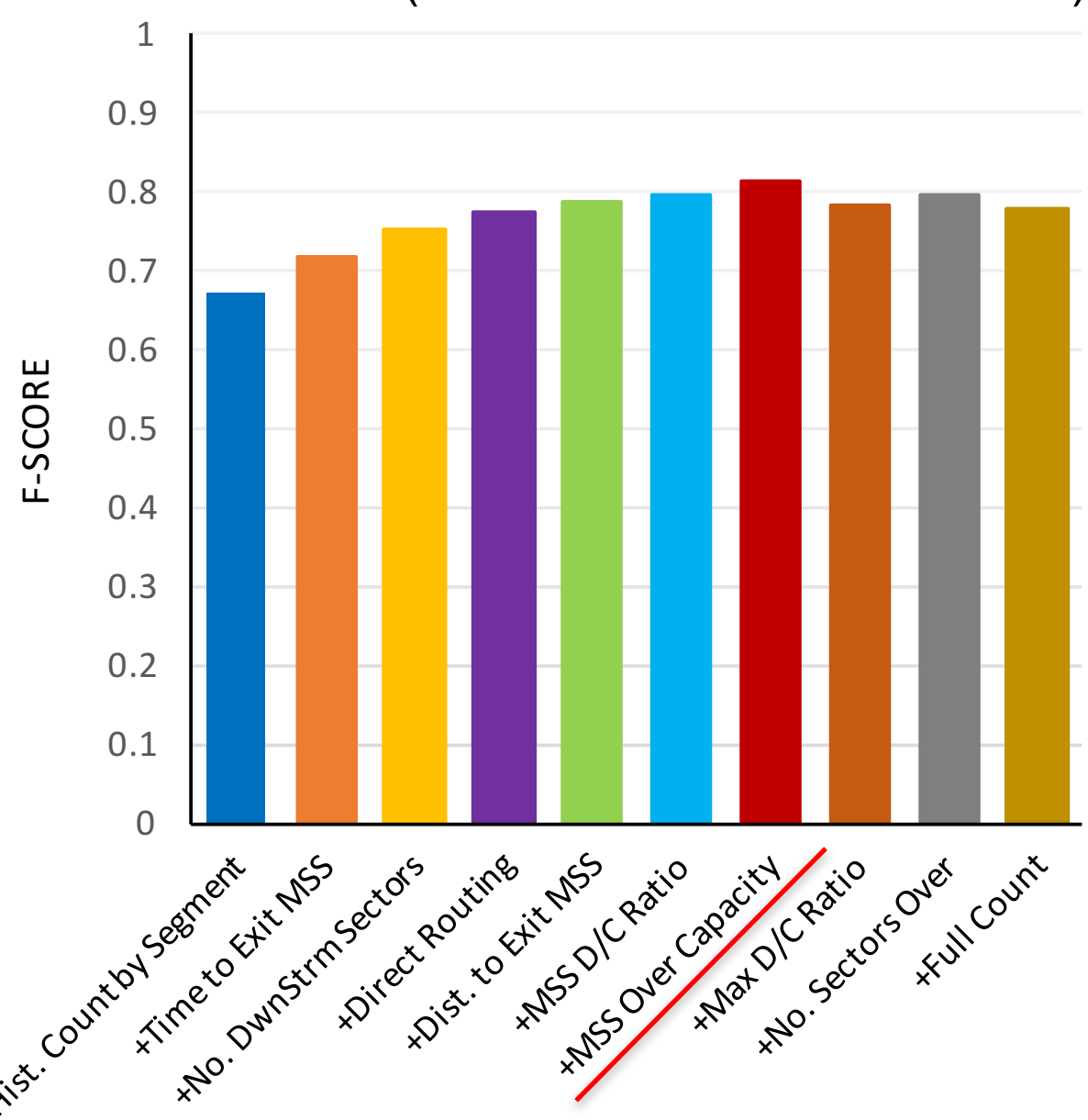

Receiver Operating Characteristic (ROC) Curve

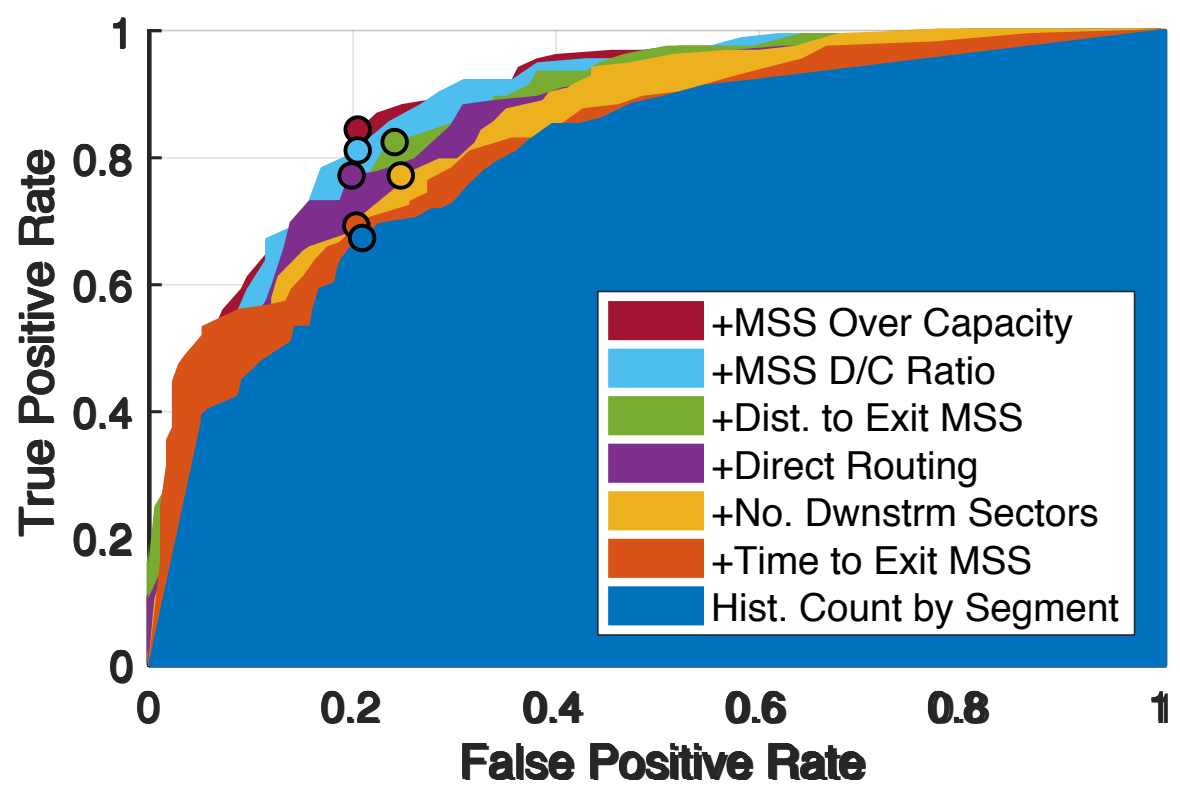




\section{Feature Selection: Forward Search}

- Method: Random Forest, 40 trees, 10-fold cross-validation

- Positive (Rejected or Modified) 40\%; Negative (Accepted) $60 \%$

- Observations: between 317 and 544, depending on features included

Model F-Score

\begin{tabular}{|c|c|c|c|c|c|c|c|c|c|c|}
\hline & $\begin{array}{c}1 \\
\text { Feature }\end{array}$ & $\begin{array}{c}2 \\
\text { Features* }\end{array}$ & $\begin{array}{c}3 \\
\text { Features }^{*}\end{array}$ & $\begin{array}{c}4 \\
\text { Features* }\end{array}$ & $\begin{array}{c}5 \\
\text { Features }^{*}\end{array}$ & $\begin{array}{c}6 \\
\text { Features* }\end{array}$ & $\begin{array}{c}7 \\
\text { Features }^{*}\end{array}$ & $\begin{array}{c}8 \\
\text { Features* }\end{array}$ & $\begin{array}{c}9 \\
\text { Features* }\end{array}$ & $\begin{array}{c}10 \\
\text { Features* }\end{array}$ \\
\hline Full Count & 0.648 & 0.695 & 0.753 & 0.771 & 0.764 & 0.766 & 0.801 & 0.775 & 0.767 & 0.780 \\
\hline Concat. Count & 0.674 & - & - & - & - & - & - & - & - & - \\
\hline Direct Routing & 0.387 & 0.597 & 0.705 & 0.775 & - & - & - & - & - & - \\
\hline No. Sectors Over & NA & 0.599 & 0.693 & 0.743 & 0.746 & 0.766 & 0.809 & 0.783 & 0.797 & - \\
\hline Max D/C Ratio & 0.255 & 0.664 & 0.751 & 0.773 & 0.769 & 0.789 & 0.772 & 0.784 & - & - \\
\hline MSS Over Capacity & NA & 0.583 & 0.674 & 0.744 & 0.758 & 0.782 & 0.815 & - & - & - \\
\hline MSS D/C Ratio & 0.381 & 0.660 & 0.749 & 0.758 & 0.773 & 0.796 & - & - & - & - \\
\hline No. Dwnstrm. Sectors & 0.484 & 0.667 & 0.755 & - & - & - & - & - & - & - \\
\hline Time to Exit MSS & 0.497 & 0.719 & - & - & - & - & - & - & - & - \\
\hline Dist. to Exit MSS & 0.467 & 0.665 & 0.719 & 0.761 & 0.789 & - & - & - & - & - \\
\hline
\end{tabular}

* Includes feature set with highest F-Score from previous column

- Feature Set with highest F-Score:

- Concatenation Count,

- Number of Downstream Sectors,

- $\quad$ Time to Exit MSS,

- Distance to Exit MSS,

- Direct Routing

- MSS Over Capacity

- MSS Demand/Capacity Ratio 


\section{Feature Selection}

- Forward Search, using a Random Forest and 10-fold cross-validation

- Feature Set with highest F-Score:

- Hist. Count by Segment,

- Number of Downstream Sectors,

- Distance to Exit Maneuver Start Sector, - Maneuver Start Sector Demand/Capacity Ratio,

- Maneuver Start Sector Over Capacity.
- Time to Exit Maneuver Start Sector,

- Direct Routing,

Receiver Operating Characteristic Curve

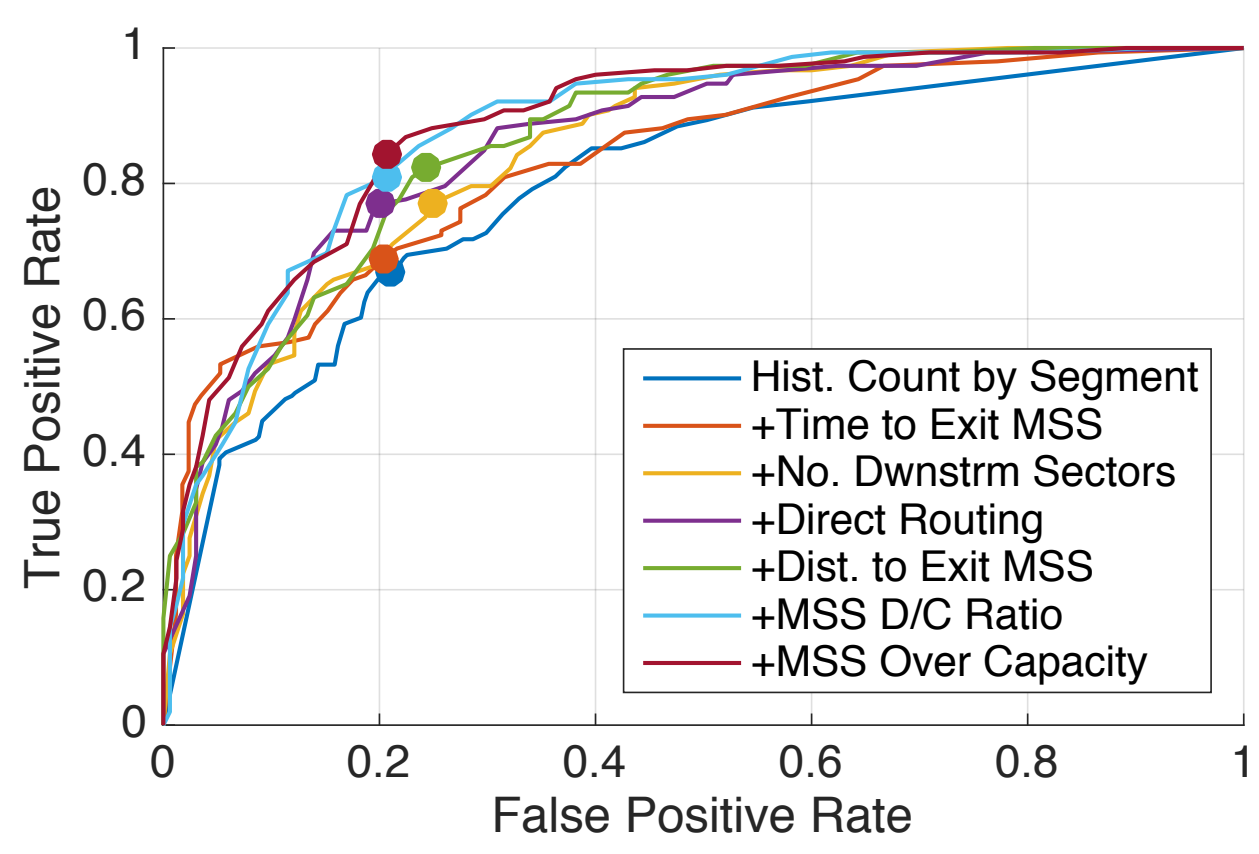




\section{Model Selection}

- 7 features

- $\quad 10$-fold cross validation

- 317 observations - 48\% Rejected (positive); 52\% Accepted (negative)

Accuracy (50\% discrimination threshold)

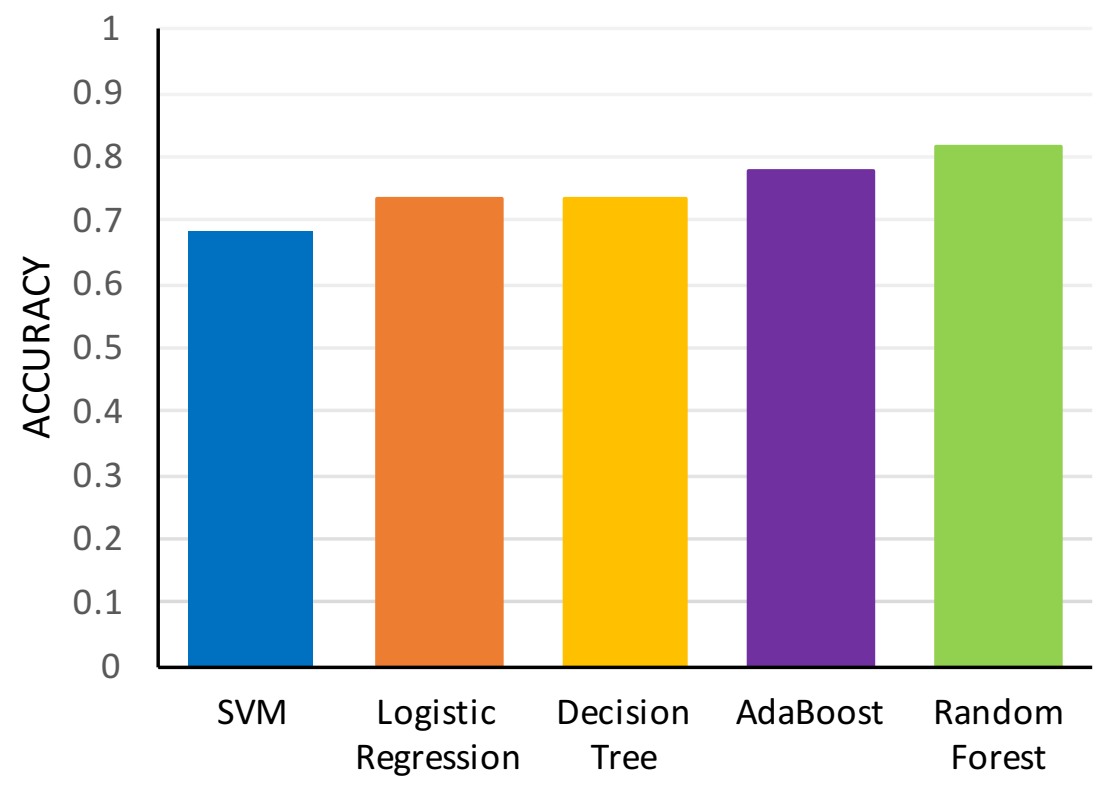

Receiver Operating Characteristic (ROC) Curve

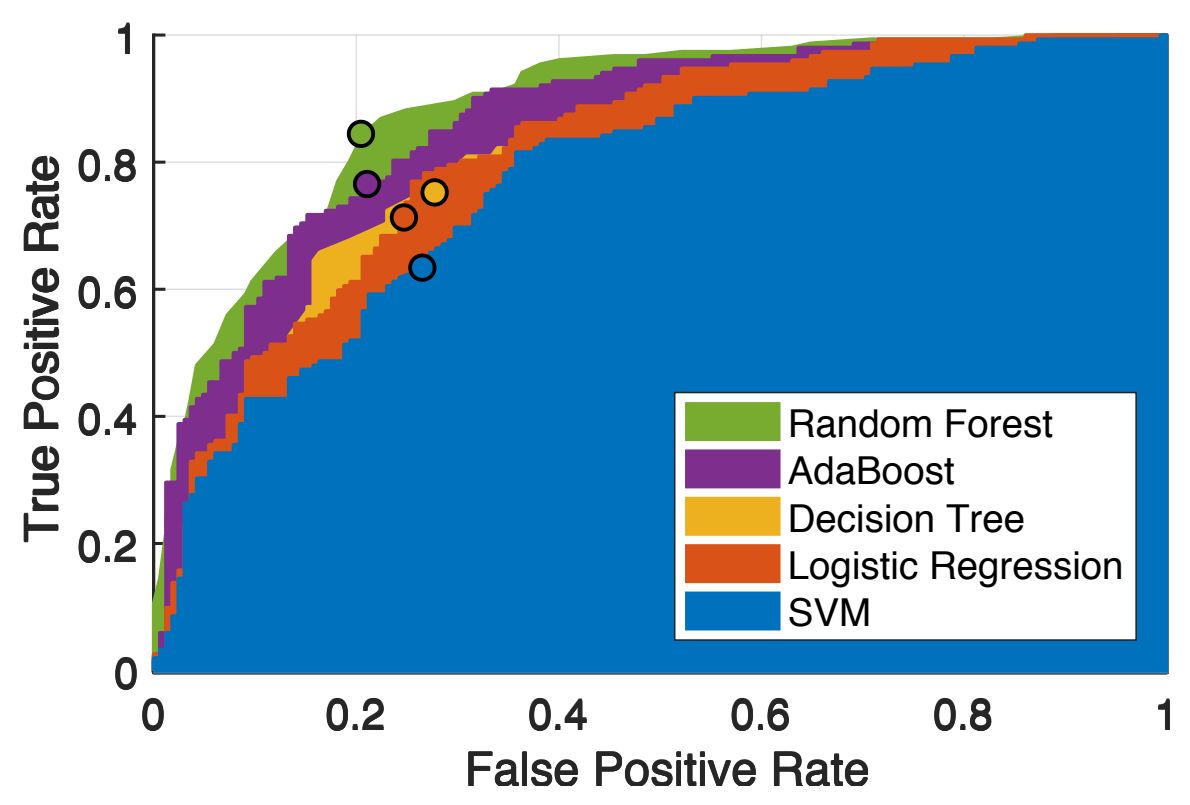

- Parameter Selection: 40 trees 


\section{Model Selection}

- 10-fold cross-validation

- 317 observations - 48\% Positive (Rejected or Modified); $52 \%$ Negative (Accepted)

\begin{tabular}{cccccc}
\hline & $\begin{array}{c}\text { Logistic } \\
\text { Regression }\end{array}$ & Decision Tree & SVM & Random Forest & AdaBoost \\
\hline Accuracy & 0.73 & 0.74 & 0.69 & 0.82 & 0.78 \\
\hline Misclassification Error & 0.27 & 0.26 & 0.31 & 0.18 & 0.22 \\
\hline True Positive Rate/Recall & 0.71 & 0.75 & 0.63 & 0.84 & 0.76 \\
\hline True Negative Rate & 0.75 & 0.72 & 0.73 & 0.79 & 0.79 \\
\hline Precision & 0.73 & 0.71 & 0.69 & 0.79 & 0.77 \\
\hline F-score & $\mathbf{0 . 7 2}$ & $\mathbf{0 . 7 3}$ & $\mathbf{0 . 6 6}$ & $\mathbf{0 . 8 2}$ & $\mathbf{0 . 7 7}$ \\
\hline
\end{tabular}

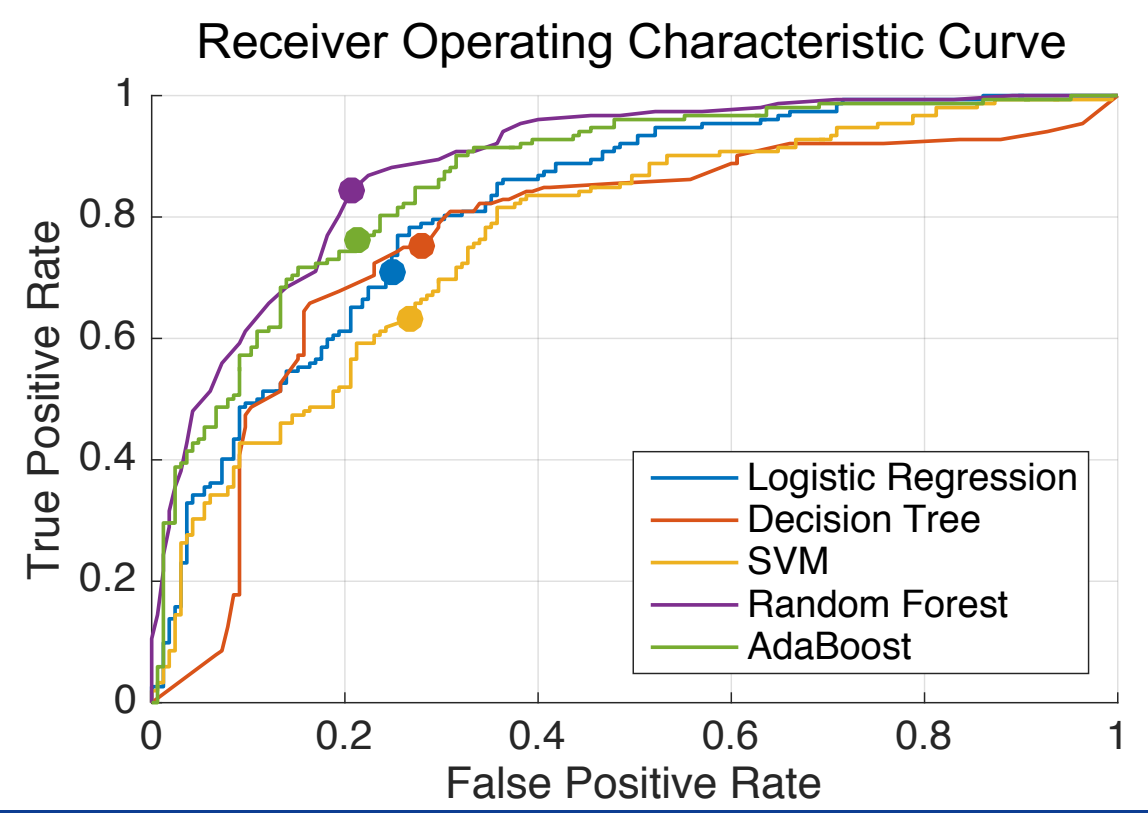




\section{Parameter Selection: Number of Weak Learners}

- Random Forest, with 10-fold cross validation

- 317 observations - 48\% Positive (Rejected or Modified); 52\% Negative (Accepted)

- Parameter value with highest F-Score: 40 trees

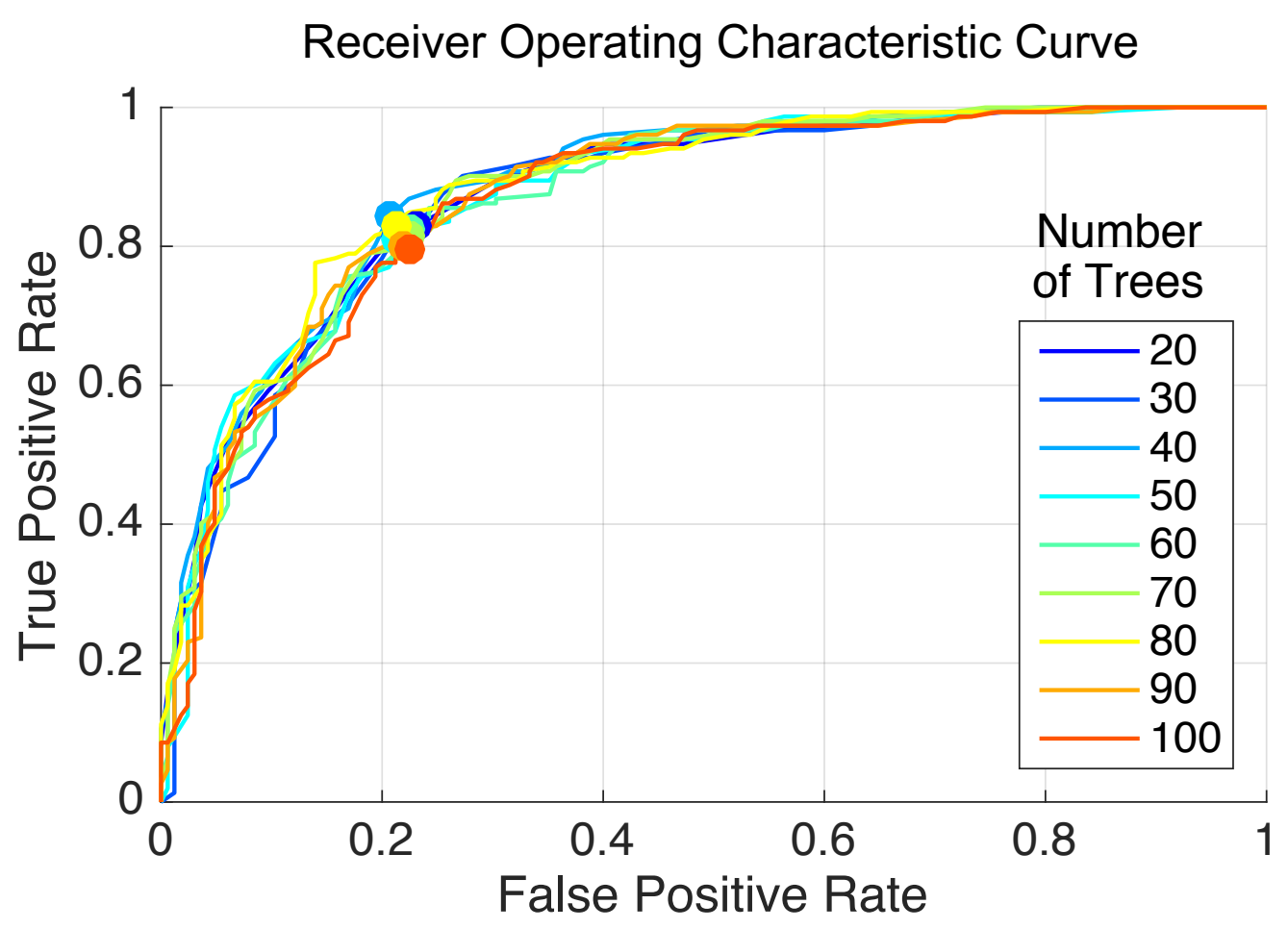




\section{Feature Selection}

- Method: Forward Search, training a Random Forest with 40 trees, using 10-fold cross-validation

- Metric: F-Score

- Observations: between 317 and 544, depending on features included

- Data Balancing: Positive (Rejected or Modified) 40\%; Negative (Accepted) $60 \%$

- Feature Set with highest F-Score (0.815):

- Hist. Count by Segment,

- Number of Downstream Sectors,

- Distance to Exit MSS,

- MSS Over Capacity
- Time to Exit MSS,

- Direct Routing

- MSS Demand/Capacity Ratio

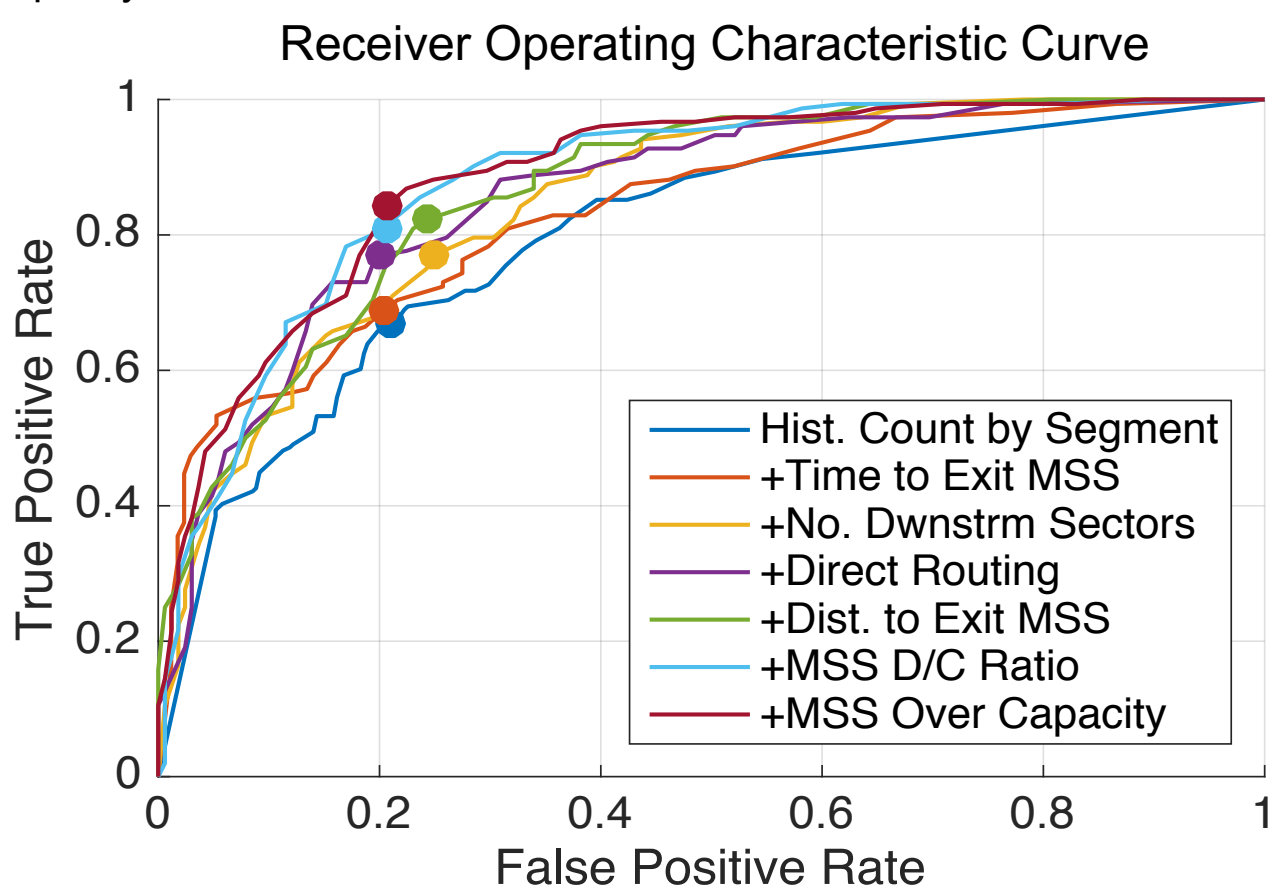




\section{Model Selection}

- $\quad$ Method: 10-fold cross-validation

- $\quad$ Observations: 317

- $\quad$ Data Balancing: Positive (Rejected or Modified) 48\%; Negative (Accepted) $52 \%$

- $\quad$ Features: Hist. Count by Segment, Time to Exit MSS, No. Downstream. Sectors, Direct Routing, Dist. to Exit MSS, MSS D/C Ratio, MSS Over Cap.

\begin{tabular}{|c|c|c|c|c|c|}
\hline & Logistic Regression & Decision Tree & SVM & Random Forest & AdaBoost \\
\hline Accuracy & 0.732 & 0.735 & 0.685 & 0.817 & 0.776 \\
\hline Misclassification Error & 0.268 & 0.265 & 0.315 & 0.183 & 0.224 \\
\hline True Positive Rate & 0.711 & 0.750 & 0.632 & 0.842 & 0.763 \\
\hline True Negative Rate & 0.752 & 0.721 & 0.733 & 0.794 & 0.788 \\
\hline Precision & 0.725 & 0.713 & 0.686 & 0.790 & 0.768 \\
\hline F-score & 0.718 & 0.731 & 0.658 & 0.815 & 0.766 \\
\hline Area Under ROC & 0.818 & 0.767 & 0.770 & 0.886 & 0.864 \\
\hline Average Precision & 0.776 & 0.687 & 0.735 & 0.870 & 0.826 \\
\hline
\end{tabular}

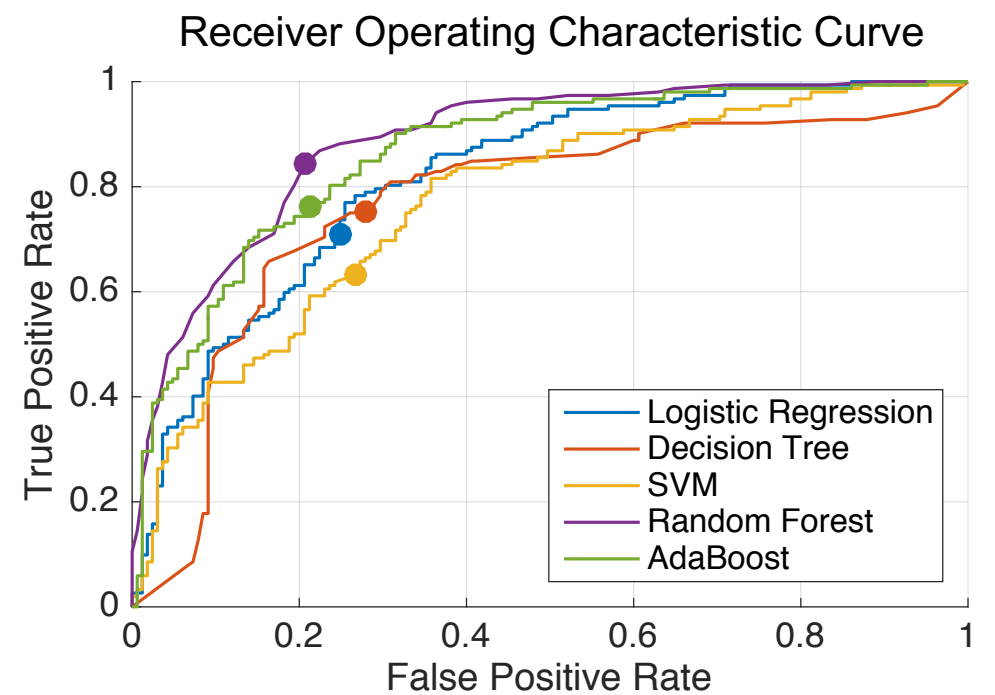




\section{Parameter Selection: Number of Weak Learners}

- $\quad$ Method: Random Forest, with 10-fold cross validation

- Data Balancing: Positive (Rejected or Modified) 48\%; Negative (Accepted) $52 \%$

Metric: F-Score

Observations: 317

- $\quad$ Features: Hist. Count By Segment, Time to Exit MSS, No. Dwnstrm. Sectors, Direct Routing, Dist. to Exit MSS, MSS D/C Ratio, MSS Over Cap.

\begin{tabular}{|c|c|c|c|c|c|c|c|c|c|}
\hline Number of Trees: & 20 & 30 & 40 & 50 & 60 & 70 & 80 & 90 & 100 \\
\hline Accuracy & 0.798 & 0.801 & 0.817 & 0.798 & 0.798 & 0.795 & 0.808 & 0.792 & 0.785 \\
\hline Misclassification Error & 0.202 & 0.199 & 0.183 & 0.202 & 0.202 & 0.205 & 0.192 & 0.208 & 0.215 \\
\hline True Positive Rate & 0.829 & 0.816 & 0.842 & 0.809 & 0.822 & 0.816 & 0.829 & 0.803 & 0.796 \\
\hline True Negative Rate & 0.770 & 0.788 & 0.794 & 0.788 & 0.776 & 0.776 & 0.788 & 0.782 & 0.776 \\
\hline Precision & 0.768 & 0.780 & 0.790 & 0.778 & 0.772 & 0.770 & 0.783 & 0.772 & 0.766 \\
\hline F-score & 0.797 & 0.797 & 0.815 & 0.794 & 0.796 & 0.792 & 0.805 & 0.787 & 0.781 \\
\hline Area Under ROC & 0.877 & 0.871 & 0.886 & 0.875 & 0.870 & 0.878 & 0.883 & 0.874 & 0.867 \\
\hline Average Precision & 0.860 & 0.820 & 0.870 & 0.833 & 0.844 & 0.854 & 0.863 & 0.840 & 0.835 \\
\hline
\end{tabular}

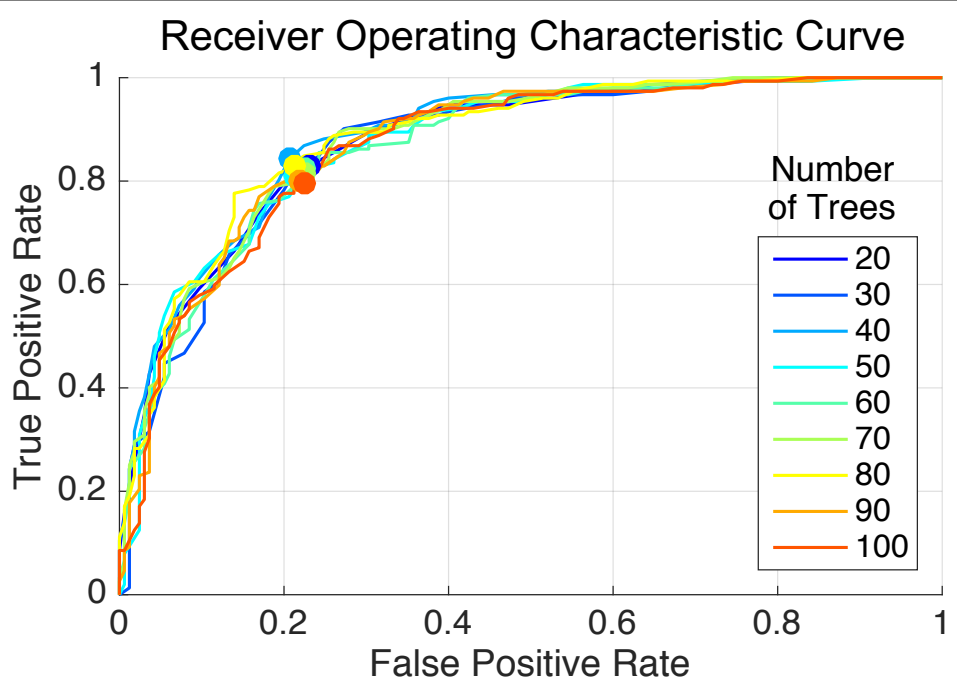




\section{Parameter Selection: Number of Weak Learners}

- Method: Random Forest, with 10-fold cross validation

- Metric: F-Score

- Observations: 317

- Data Balancing: Positive (Rejected or Modified) 48\%; Negative (Accepted) $52 \%$

- Features: Hist. Count by Segment, Time to Exit MSS, No. Downstream Sectors, Direct Routing, Dist. to Exit MSS, MSS D/C Ratio, MSS Over Cap.

- Parameter value with highest F-Score (0.815):

- 40 trees

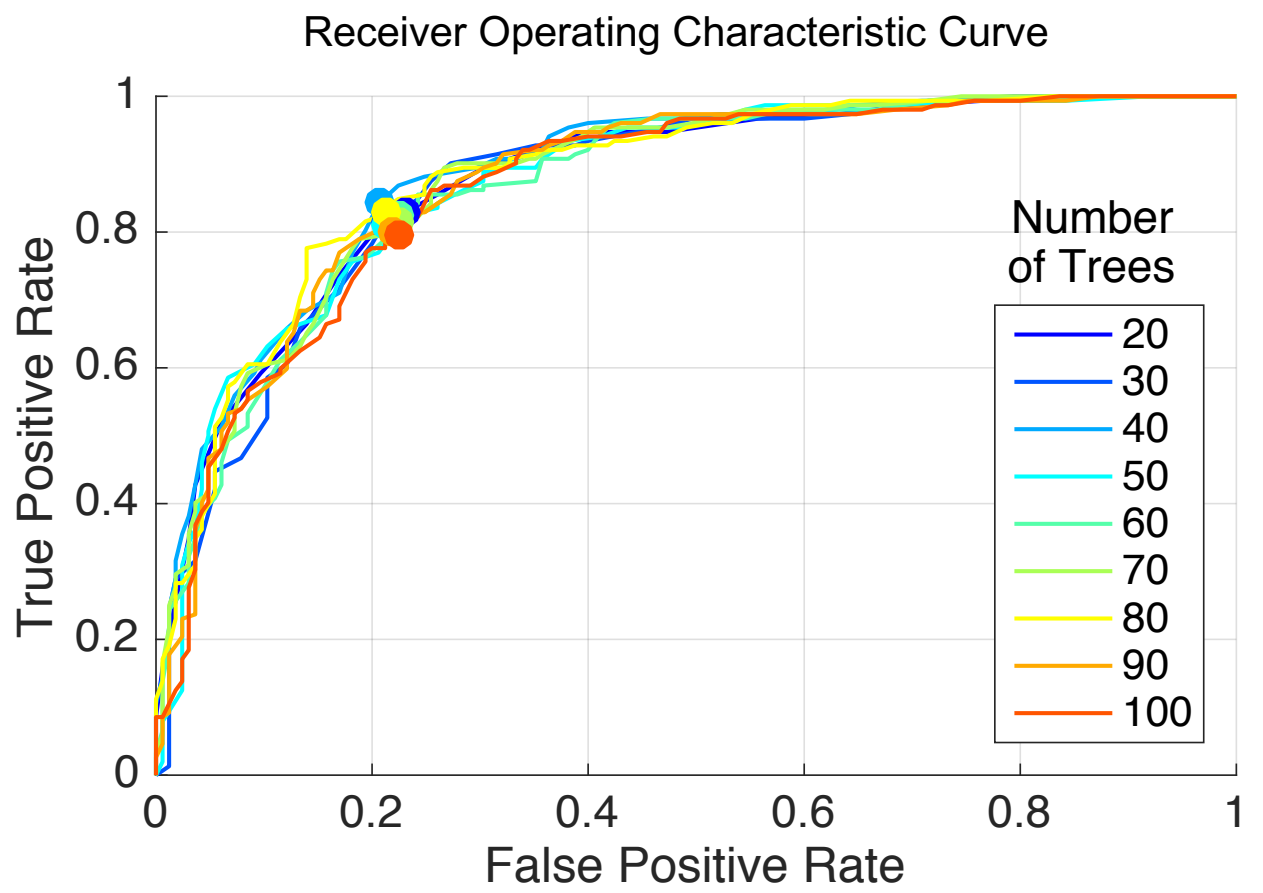




\section{Model Validation}

- Method: Random Forest, with 10-fold nested cross validation

- Observations: 317

- Data Balancing: Positive (Rejected or Modified) 40\%; Negative (Accepted) $60 \%$

\begin{tabular}{cc}
\hline & $\begin{array}{c}\text { Nested Cross- } \\
\text { Validation }\end{array}$ \\
\hline Accuracy & 0.744 \\
\hline Misclassification Error & 0.256 \\
\hline True Positive Rate/Recall & 0.875 \\
\hline True Negative Rate & 0.624 \\
Precision & 0.682 \\
FScore & $\mathbf{0 . 7 6 7}$ \\
Area Under ROC & 0.814 \\
\hline Average Precision & 0.742 \\
\hline
\end{tabular}

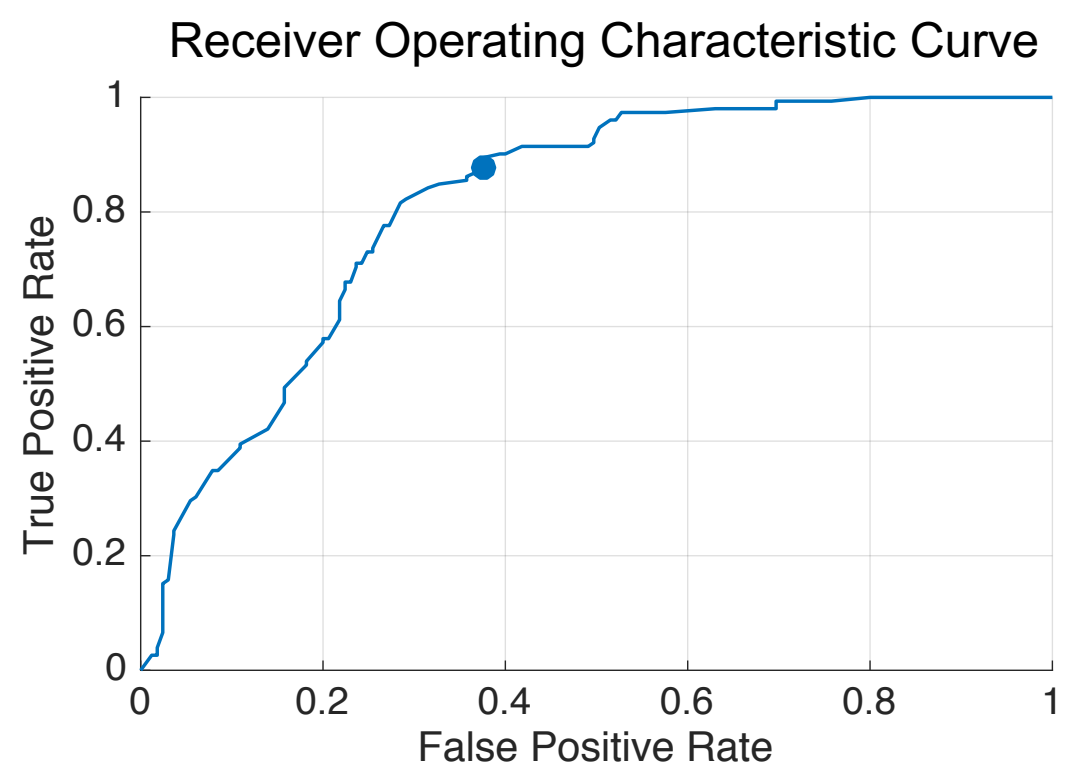




\section{Comparison to One-Class Classification}

- Method: 10-fold cross-validation

- Observations: 317

- Data balancing: Positive (Rejected or Modified) 48\%; Negative (Accepted) $52 \%$

- $\quad$ Features: Hist. Count by Segment, Time to Exit MSS, No. Downstream Sectors, Direct Routing, Dist. to Exit MSS, MSS D/C Ratio, MSS Over Capacity

\begin{tabular}{cccc}
\hline & Random Forest & Two-Class SVM & One-Class SVM \\
\hline Accuracy & 0.817 & 0.685 & 0.558 \\
\hline Misclassification Error & 0.183 & 0.315 & 0.442 \\
True Positive Rate/Recall & 0.842 & 0.632 & 0.211 \\
\hline True Negative Rate & 0.794 & 0.733 & 0.879 \\
Precision & 0.790 & 0.686 & 0.615 \\
\hline FScore & $\mathbf{0 . 8 1 5}$ & $\mathbf{0 . 6 5 8}$ & $\mathbf{0 . 3 1 4}$ \\
\hline
\end{tabular}

